\title{
ON THE EXISTENCE OF NORMAL ANALYTIC FUNCTIONS
}

\author{
PENTTI JÄRVI
}

1. It has been asked (Pommerenke [1. p. 169], Minda [4, p. 119]) whether there are Riemann surfaces which do not carry nonconstant normal meromorphic functions. The purpose of the present note is to exhibit an open Riemann surface which does not tolerate nonconstant normal analytic functions. Our construction is a modification of an example by Royden [5, p. 6]. By the way, we shall find how to produce Myrberg-type surfaces with the property that each normal analytic function possesses a limit at the (single) parabolic ideal boundary element. An example of this sort has recently been given by Harju [2]; the construction there makes explicit use of uniformization.

2. Let $S$ stand for the complex plane $C$ less $\{0\}$ and the slits $\left[1 / 2^{2 n}, 1 / 2^{2 n-1}\right]$, $\left[2^{2 n-1}, 2^{2 n}\right], n \in N$. Then $W^{\prime}$, a two-sheeted branched covering surface of $C \backslash\{0\}$, is obtained by joining two copies $S_{1}$ and $S_{2}$ of $S$, the "upper" and the "lower" sheet of $W^{\prime}$, identifying in the usual manner the upper edges of the slits of $S_{1}$ with the corresponding lower edges of the slits of $S_{2}$ and vice versa. Let $f_{0}$ denote the natural projection $W^{\prime} \rightarrow C$. Note that $f_{0}^{-1}(\{|z|=1\})$ consists of two Jordan curves $C_{1}^{\prime} \subset S_{1}$ and $C_{2}^{\prime} \subset S_{2}$.

Now let $\alpha$ be an irrational multiple of $2 \pi$. Cut $W^{\prime}$ along $C_{1}^{\prime}$ and denote by $B_{1}$ and $B_{2}$ the boundary curves of the bordered surface obtained corresponding to the parts $f_{0}^{-1}(\{|z|<1\})$ and $f_{0}^{-1}(\{|z|>1\})$, respectively. Next identify $p \in B_{1}$ with $q \in B_{2}$, provided $f_{0}(p)=e^{i \alpha} f_{0}(q)$. The result is a Riemann surface, say $W$, and we claim that $W$ has the property mentioned before.

3. Denote by $C \subset W$ the image of $B_{1}$ (or $B_{2}$ ) under the identification, and let $\theta \mapsto \omega(\theta)$ be the parametrization of $C$ induced by the mapping $e^{i \theta} \mapsto f_{0}^{-1}\left(e^{i \theta}\right) \in B_{1}$. Note that $f_{0}$ can be taken as defined (and analytic) on $W \backslash C$ by the identification of $W^{\wedge} C_{1}^{\prime}$ with $W \backslash C$. Set $W_{1}=W \cap f_{0}^{-1}(\{|z|<1\})$ and $W_{2}=W \cap f_{0}^{-1}(\{|z|>1\})$. Denote by $\varphi$ the homothetic transformation $z \mapsto z / 4, \quad z \in D^{*}=\{z \in C|0<| z \mid<1\}$. It is clear that $\varphi$ induces an analytic mapping $\psi: W_{1} \rightarrow W_{1}$ such that $f_{0} \circ \psi=\varphi \circ f_{0}$ on $W_{1}$ (just send the points of $S_{i}$ into $S_{i}, i=1,2$, and note that $\varphi$ preserves the slits).

Suppose $f$ is a normal analytic function on $W$. Then, by the principle of hyperbolic metric, $g=f \mid W_{1}$ is normal on $W_{1}$. This means that there is a positive constant 
$m$ such that

$$
\frac{d s_{g}(p)}{d \sigma(p)} \leqq m \text { for } p \in W_{1},
$$

where $d \sigma(p)$ denotes the hyperbolic metric at $p$ inherited from the universal covering surface, i.e., the unit disc, and $d s_{g}(p)=\left|g^{\prime}(z)\right||d z| /\left(1+|g(z)|^{2}\right)$ in terms of any local coordinate $z$ at $p$ [3]. The least upper bound in (1) is called the order of normality of $g$. For the sake of reference, we state a simple lemma.

Lemma. Let $W$ be a Riemann surface whose universal covering surface is hyperbolic. Suppose $\mathscr{F}$ is a family of normal meromorphic functions on $W$ such that the orders of normality of functions in $\mathscr{F}$ are uniformly bounded. Then $\mathscr{F}$ is a normal family.

Proof. Suppose $d s_{f}(p) / d \sigma(p) \leqq m$ for all $p \in W$ and for all $f \in \mathscr{F}$. It follows that $\mathscr{F}$ is equicontinuous on compact subsets of $W$. The assertion is now a direct consequence of the Ascoli-Arzela theorem.

We will apply the above Lemma in the situation $W=W_{1}$ and $\mathscr{F}=$ $\left\{g \circ \psi^{n} \mid n \in N\right\}$ (here $\psi^{n}$ denotes the $n$-th iterate of $\psi$ ). That $\mathscr{F}$ is normal follows from the normality of $g$ and from the principle of hyperbolic metric. Note that the orders of normality of $g \circ \psi^{n}$ are bounded by that of $g$.

4. We are going to show that $g$ admits a representation

$$
g=h \circ\left(f_{0} \mid W_{1}\right),
$$

where $h$ is meromorphic in the disc $D=\{z \in C|| z \mid<1\}$. This of course implies that $g$ possesses a limit at the ideal boundary point $\beta_{0}$ lying "over" the point $0: \lim _{p \rightarrow \beta_{0}} g(p)=$ $h(0)$. First suppose that $g$ has only a finite number of zeros in $W_{1}$. Then there is $n_{0} \in N$ such that $g$ does not vanish in $f_{0}^{-1}\left(\left\{0<|z| \leqq(1 / 4)^{n} 0\right\}\right)$. Set $\mathscr{F}_{n_{0}}=\left\{g \circ \psi^{n} \mid n \geqq n_{0}\right\}$. Then $\mathscr{F}_{n_{0}}$ is a normal family of analytic functions on $W_{1}$. Therefore we may pick out a convergent subsequence $\left(g_{n_{k}}=g \circ \psi^{n_{k}}\right)$. The limit function $g_{0}$ is either analytic on $W_{1}$ or identically equal to $\infty$.

Assume that $g_{0}$ is analytic. Then $\left\{g_{n_{k}} \mid k \in N\right\}$ is uniformly bounded on the Jordan curve $F_{0}=f_{0}^{-1}(\{|z|=\sqrt{2} / 4\})$ (note that $F_{n}=f_{0}^{-1}\left(\left\{|z|=\sqrt{2} / 2^{2(n+1)}\right\}\right)$ is a Jordan curve which separates $\beta_{0}$ from $f_{0}^{-1}\left(\left\{\sqrt{2} / 2^{2(n+1)}<|z|<1\right\}\right)$ for each $\left.n\right)$. Hence there is $M>0$ such that $|g(p)| \leqq M$ for each $p \in \cup_{k=1}^{\infty} F_{n_{k}}$. By the maximum modulus principie, $g$ is bounded by $M$ in $f_{0}^{-1}\left(\left\{\sqrt{2} / 2^{2 n_{k+1}} \leqq|z| \leqq \sqrt{2} / 2^{2 n_{k}}\right\}\right)$ for each $k \in N$. Hence $g$ is bounded in $W_{1}$. It is now readily seen by the well-known argument due to P. J. Myrberg (see e.g. [5, p. 6]) that $g=h \circ\left(f_{0} \mid W_{1}\right)$ for some function $h$ analytic on $D$.

Then assume that $g_{0} \equiv \infty$. Since $g$ has no zeros in $f_{0}^{-1}\left(\left\{0<|z|<(1 / 4)^{n_{0}}\right\}\right)$, $1 / g_{n_{k}}$ is analytic on $W_{1}$ for each $k \in N$. Clearly, $1 / g_{n_{k}} \rightarrow 0$ uniformly in compact parts of $W_{1}$ as $k \rightarrow \infty$. By the argument cited above, $1 / g=h \circ\left(f_{0} \mid W_{1}\right)$ with $h$ analytic on $D$, whence $g=(1 / h) \circ\left(f_{0} \mid W_{1}\right)$.

There remains the case that the number of the zeros of $g$ is infinite. Let $\left(p_{k}\right)$ be an 
enumeration of the zeros of $g$ in $f_{0}^{-1}(\{0<|z|<1 / 16\})$. For each $k \in N$ there is a unique $q_{k} \in f_{0}^{-1}(\{1 / 16 \leqq|z|<1 / 4\})$ and a unique $n_{k} \in N$ such that $\psi^{n_{k}}\left(q_{k}\right)=p_{k}$. Passing to a subsequence of $\left(p_{k}\right)$, we may assume that $n_{1}<n_{2}<\ldots$. Again, $\left\{g_{n_{k}}=g \circ \psi^{n_{k}} \mid k \in N\right\}$ is a normal family of functions analytic on $W_{1}$, and we may choose a convergent subsequence $\left(g_{n_{k_{j}}}\right)$. Clearly, each $g_{n_{k_{j}}}$ has a zero in the compact set $f_{0}^{-1}(\{1 / 16 \leqq|z| \leqq 1 / 4\}) \subset W_{1}$. Hence the limit $g_{0}=\lim _{j \rightarrow \infty} g_{n_{k_{j}}}$ must be analytic on $W_{1}$. Reasoning as before, we see that $g=h \circ\left(f_{0} \mid W_{1}\right)$ with $h$ bounded in $D$.

5. Of course, the state of affairs is similar in $W_{2}: f \mid W_{2}=h \circ\left(f_{0} \mid W_{2}\right)$ with $h$ meromorphic on $\{z \in \hat{C}=C \cup\{\infty\}|| z \mid>1\}$. Now let $\left(p_{n}^{\prime}\right)$ be a sequence of points in $S_{1} \cap W_{1}$ and $\left(p_{n}^{\prime \prime}\right)$ a sequence of points in $S_{1} \cap W_{2}$ such that $\lim _{n \rightarrow \infty} p_{n}^{\prime}=\lim _{n \rightarrow \infty} p_{n}^{\prime \prime}=$ $=p\left(\in W^{\prime}\right)$ with $f_{0}(p)=e^{i \theta}$. Further, let $\left(q_{n}^{\prime}\right)$ and $\left(q_{n}^{\prime \prime}\right)$ be sequences of points in $S_{2} \cap W_{1}$ and $S_{2} \cap W_{2}$, respectively, such that $f_{0}\left(p_{n}^{\prime}\right)=f_{0}\left(q_{n}^{\prime}\right)$ and $f_{0}\left(p_{n}^{\prime \prime}\right)=f_{0}\left(q_{n}^{\prime \prime}\right)$ for each $n$. Clearly, $\lim _{n \rightarrow \infty} q_{n}^{\prime}=\lim _{n \rightarrow \infty} q_{n}^{\prime \prime}=q$ with $f_{0}(q)=e^{i \theta}$. By the construction of $W$, $\lim _{n \rightarrow \infty} f\left(p_{n}^{\prime}\right)=f(\omega(\theta))$ and $\lim _{n \rightarrow \infty} f\left(p_{n}^{\prime \prime}\right)=f(\omega(\theta+\alpha))$. On the other hand, $\lim _{n \rightarrow \infty} f\left(q_{n}^{\prime}\right)=\lim _{n \rightarrow \infty} f\left(q_{n}^{\prime \prime}\right)=f(q)$. The above factorization in turn implies $f\left(p_{n}^{\prime}\right)=$ $f\left(q_{n}^{\prime}\right)$ and $f\left(p_{n}^{\prime \prime}\right)=f\left(q_{n}^{\prime \prime}\right)$ for each $n$. Hence $f(\omega(\theta))=f(\omega(\theta+\alpha))$. It follows, by the choice of $\alpha$, that $f$ assumes the value $f(\omega(\theta))$ in a set dense in $C$. Thus $f$ must be constant. We have thereby established the following

Proposition. There exist open Riemann surfaces which do not carry nonconstant normal analytic functions.

Remarks. (1) The reader will have noticed that the reasoning in Section 4 is an adaptation of one of the classical proofs of the "big" Picard theorem.

(2) It is clear from the above discussion that the original question of Pommerenke can be answered in the same direction, provided one can exhibit a Myrberg-type, say two-sheeted, surface $W$ lying over $D^{*}$ such that each normal meromorphic function on $W$ admits a factorization of type (2).

(3) As noted before, surfaces like $W_{1}$ have been constructed by means of Fuchsian groups [2, pp. 16-35]. An essential feature of $W_{1}$, as given above, is that it possesses enough symmetry to admit useful analytic self-mappings. Obviously, the number of the sheets plays an inessential role in the construction. 


\section{References}

[1] Clunie, J., and W. K. Hayman (eds): Symposium on Complex Analysis, Canterbury, 1973, London Mathematical Society Lecture Note Series 12, Cambridge University Press, Cambridge, 1974.

[2] HARJU, J.: Absolute branch points on Riemann surfaces. - Ann. Acad. Sci. Fenn. Ser. A I Math. Dissertationes 51, 1984, 1-36.

[3] LeHTo, O., and K. I. VIRTANEN: Boundary behaviour and normal meromorphic functions. - Acta Math. 97, 1957, 47-65.

[4] MindA, C. D.: Marden constants for Bloch and normal functions. - J. Analyse Math. 42, 1982/83, $117-127$.

[5] Royden, H. L.: Open Riemann surfaces. - Ann. Acad. Sci. Fenn. Ser. A I Math. 249/5, 1958, $1-13$.

University of Helsinki

Department of Mathematics

SF-00100 Helsinki

Finland

Received 11 December 1984 